\title{
Schweizer Landwirtschaft vor der Marktöffnung : Skepsis und Reaktionen
}

\section{Gérard Vuffray}

\section{(2) OpenEdition}

1 Journals

\section{Electronic version}

URL: http://journals.openedition.org/sjep/869

DOI: 10.4000/sjep.869

ISSN: 1663-9677

\section{Publisher}

Institut de hautes études internationales et du développement

\section{Printed version}

Date of publication: 1 mai 2002

Number of pages: $27-38$

ISSN: 1660-5926

\section{Electronic reference}

Gérard Vuffray, "Schweizer Landwirtschaft vor der Marktöffnung : Skepsis und Reaktionen »,

Schweizerisches Jahrbuch für Entwicklungspolitik [Online], 21 | 2002, Online erschienen am: 10

September 2012, abgerufen am 08 September 2020. URL : http://journals.openedition.org/sjep/869

DOI : https://doi.org/10.4000/sjep.869 


\section{SCHWEIZER LANDWIRTSCHAFT VOR DER MARKTÖFFNUNG: SKEPSIS UND REAKTIONEN}

GÉrARD VufFraY*

\section{DIE SCHWEIZ: EINER DER WELTWEIT OFFENSTEN MÄRKTE FÜR AGRARPRODUKTE UND NAHRUNGSMITTEL}

Je nach Jahr kann die Schweizer Landwirtschaft zwischen 58 und $62 \%$ des heimischen Agrargüterbedarfs abdecken. Damit besitzt die Schweiz im Nahrungsmittelbereich zum einen den weltweit niedrigsten Selbstversorgungsgrad und zum anderen den offensten Markt für die Einfuhr von Agrargütern und Nahrungsmitteln. Dieser Sachverhalt ist von entscheidender Bedeutung und verdient es, eingangs erwähnt zu werden, da er der gängigen Auffassung widerspricht und in der Debatte um die künftige Entwicklung der Marktöffnung - sei es in bilateralen Verhandlungen mit verschiedenen Ländern, im Rahmen der WTO-Verhandlungsrunden oder in der Diskussion um die vollständige Liberalisierung der Agrarimporte aus den ärmsten Ländern der Welt - in der Regel untergeht.

In der Frage um die Marktöffnung sollte diese Tatsache stets betont werden, zumal die meisten anderen Länder - nicht einmal die eifrigsten Verfechter des Freihandels wie etwa die USA, die Europäische Union oder die Länder der Cairns-Gruppe - sich kaum damit abfinden dürften, dass durchschnittlich $40 \%$ ihres Nahrungsmittelbedarfs durch Importe abgedeckt werden müssen. Im Falle der Schweiz liegt der Selbstversorgungsgrad je nach Produktgruppe zwischen 18\% (Fette und pflanzliche Öle) und $111 \%$ (Milchprodukte). Beim Gemüse beträgt er $52 \%$, beim Getreide $62 \%$ und bei der Fleischproduktion insgesamt $70 \% 1$.

\section{DIE BESONDEREN RAHMENBEDINGUNGEN DER SCHWEIZER LANDWIRTSCHAFT}

Zumindest in zwei Punkten unterscheiden sich die Rahmenbedingungen der Schweizer Landwirtschaft wesentlich von jenen der meisten anderen Länder. Diese beiden Aspekte und die damit verbundenen Sachzwänge sollten bei der Frage nach der Öffnung der Märkte für Importe ebenfalls berücksichtigt werden :

a Bei der Schweizer Landwirtschaft handelt es sich im Wesentlichen um eine Bergbauernwirtschaft, denn mehr als die Hälfte der landwirtschaftlich genutzten Flächen liegt über $1000 \mathrm{~m}$. In diesen Lagen herrschen schwierige topografische und klimatische Bedingungen, und die Auflagen und Kontrollen im Hinblick auf den Umweltschutz, die Produktionsmethoden und die Viehhaltung sind in der Regel strenger als anderswo.

- Die Schweizer Landwirtschaft ist eng in den wirtschaftlichen Hintergrund des Landes eingebunden. Die Produktionskosten sind in hohem Masse von den

* Agronomingenieur, Geschäftsleiter von Uniterre.

1. Bundesamt für Landwirtschaft, Agrarbericht 2000 und 2001, Bern. 
Kosten der übrigen Produktionsfaktoren in der Schweiz abhängig, die zu den weltweit höchsten zählen: Hohe Land-, Bau- und Maschinenpreise und hohe Dienstleistungs-, Arbeits-, Energie- und Transportkosten, hohe Düngerpreise, Versicherungsprämien und so weiter ${ }^{2}$. Die Verkaufspreise der produzierten Güter werden massgeblich von den hohen wirtschaftlichen und sozialen Kosten in der Schweiz bestimmt.

Diese Faktoren verteuern die Schweizer Landwirtschaftsprodukte entscheidend und werden über sämtliche Verarbeitungs- und Handelsschritte hinweg auf die Konsumenten abgewälzt. Ungeachtet des Know-how und der Produktivität der Schweizer Bauern sind deshalb Schweizer Agrarerzeugnisse immer erheblich teurer als zahlreiche ausländische Produkte.

Damit lässt sich, wenn auch vereinfacht, die Grundproblematik zusammenfassen, mit der die Schweizer Bauern im Hinblick auf eine weiter gehende Öffnung des Marktes konfrontiert sind. Darüber hinaus ist die schweizerische Landwirtschaft seit einigen Jahren aber auch verpflichtet, einen gewissen Service public zu erbringen.

\section{MULTIFUNKTIONALITÄT UND NACHHALTIGKEIT}

Während mehr als zehn Jahren war die Landwirtschaft Thema intensiver politischer Diskussionen und mehrerer eidgenössischer Abstimmungen. Im Zuge dieses Prozesses wurden eine Verfassungsänderung beschlossen, ein neues Landwirtschaftsgesetz verabschiedet, sämtliche damit zusammenhängenden Gesetze und Verordnungen umfassend revidiert und mithin die Rolle der Landwirtschaft und die Vergütung der erbrachten Leistungen von Grund auf neu definiert. Da es der marktwirtschaftlichen Philosophie zuwider läuft, wenn sich die ökologischen, sozialen und kulturellen Kosten der Agrarproduktion in den Preisen niederschlagen, mussten die Kosten für landwirtschaftliche Produkte in zwei Komponenten aufgegliedert werden, von denen die eine zu Lasten der Allgemeinheit geht und die andere von den Konsumenten bezahlt wird. Unter dem Druck, der namentlich vom GATT und den zwischen 1989 und 1995 geführten internationalen Verhandlungen ausging, wurden die Konzepte der Multifunktionalität und der nachhaltigen Entwicklung präzisiert und vom Marktwert der Agrargüter losgelöst.

Der neue Artikel 104 der Bundesverfassung gesteht der Landwirtschaft beim Schutz der natürlichen Lebensgrundlagen und der Pflege der Kulturlandschaft, aber auch bei der Erhaltung der Bevölkerung und der Bodenbewirtschaftung in ländlichen Gebieten - immerhin rund die Hälfte des Territoriums der Schweiz eine zentrale Rolle zu. Daneben verankert er den wichtigsten, ebenfalls «nichtkommerziellen» Auftrag der Landwirtschaft, nämlich die Sicherstellung der Nahrungsmittelversorgung der Bevölkerung. Die so genannten «nicht-kommerziellen » Leistungen der Landwirtschaft werden seither über Direktzahlungen des Bundes an die Landwirte abgegolten. Diese Direktzahlungen basieren auf einem komplexen Katalog von Leistungen und Verpflichtungen, die auf freiwilliger oder auf vertraglicher Basis geregelt und von den Kantonen laufend überprüft werden. Diese Leistungen werden grundsätzlich getrennt vom Marktwert der verkauften Produkte verrechnet.

2. Schweizerischer Bauernverband, Possibilités de réduire les coûts de production dans l'agriculture, 1996. 
Diese Neudefinition der Rolle der Landwirtschaft hat in gewisser Weise zwei voneinander getrennte Märkte und zwei unterschiedliche Kundensegmente hervorgebracht: Einerseits die Konsumenten, die Nahrungsmittel kaufen, und andererseits der Bund, der eine Reihe von Dienstleistungen erwirbt, darunter die Nahrungsmittelversorgung des Landes, die Erhaltung der natürlichen Lebensgrundlagen, die dezentrale Besiedlung des Landes und die Pflege der Kulturlandschaft. Der Preis, den die von der Landwirtschaft bereitgestellten Güter und Dienstleistungen auf ihrem jeweiligen Markt erzielen, wird bestimmt durch den Lebensstandard und die Produktionskosten in der Schweiz, aber auch durch die Ansprüche der jeweiligen Kundschaft u.a.m.

Die Ziele im Hinblick auf die Besiedlung ländlicher Gebiete, die Landschaftspflege und die Erhaltung der natürlichen Lebensgrundlagen lassen sich häufig nur schwer definieren, und ihre Verwirklichung durch konkrete Massnahmen und im Rahmen zeitlich begrenzter und garantierter Verträge gestaltet sich oft komplex. Über den wirtschaftlichen Wert «nicht-kommerzieller» Leistungen liesse sich lange debattieren: Welches ist der gerechte Preis für die 7 bis $10 \%$ landwirtschaftlichen Ausgleichsflächen, die für die Nahrungsmittelproduktion nicht mehr genutzt werden können, weil sie der Erhaltung der Artenvielfalt in ländlichen Gebieten und dem Schutz von Flora und Fauna dienen? Welches ist der gerechte Preis dafür, dass gewisse Landschaften geschützt und in einen naturnahen Zustand zurückversetzt werden? Welches ist der gerechte Preis für Eier von Hühnern aus Freilandhaltung, für Fleisch, das ohne Hormone, Antibiotika oder gentechnisch veränderte Organismen produziert wurde, oder schlicht für die Erhaltung der Landwirtschaft in der Schweiz?

\section{DIE QUADRATUR DES KREISES}

Die Schweizer Landwirtschaft steht heute vor einer Herausforderung, die der Quadratur des Kreises gleichkommt, denn von ihr wird verlangt, gleichzeitig mehrere einander widersprechende verfassungsmässige Ziele zu verfolgen: Sie soll ihre Wettbewerbsfähigkeit steigern und dennoch ökologische Aspekte verstärkt berücksichtigen, und sie soll rationeller und kostengünstiger produzieren, während die Agrargüterpreise und das landwirtschaftliche Einkommen sinken und gleichzeitig die Umweltvorgaben verschärft werden. Über 20 '000 landwirtschaftliche Betriebe sind innerhalb von 10 Jahren verschwunden - ein Rückgang von $25 \%$. In den kommenden Jahren dürfte sich dieser Prozess noch beschleunigen. Das Durchschnittseinkommen der verbleibenden Landwirte stagniert nach wie vor bei rund 3000 Franken monatlich (inklusive Direktzahlungen), das sind je nach Region zwischen 35 und $55 \%$ weniger als das Einkommen, welches vergleichbar qualifizierte Erwerbstätige in anderen Sektoren erzielen ${ }^{3}$.

In Anbetracht dessen kann es nicht verwundern, dass der anhaltende Druck für eine weitere Öffnung für Importe in Bauernkreisen auf wenig Gegenliebe stösst, zumal die ausländischen Erzeugnisse den für Schweizer Produkte geltenden Umwelt- und Qualitätsanforderungen grösstenteils nicht genügen. Dabei ist unbestritten, dass der Preis der meisten importierten Nahrungsmittel nur einen

3. FAT, Die wirtschaftliche Entwicklung der schweizerischen Landwirtschaft 1990 bis 1999, Bericht Nr. $556,2000$. 
Bruchteil der Produktionskosten der schweizerischen Erzeugnisse beträgt und dass die einheimische Nahrungsmittelproduktion ohne einen adäquaten Schutz keinerlei Zukunftschancen hat.

\section{DEVALORISIERUNG DER LANDWIRTSCHAFTLICHEN ARBEIT}

Für den Durchschnittsbürger scheint es heute selbstverständlich, importierte Güter zu möglichst niedrigen Preisen zu kaufen, wodurch das Einkommen der Bauern für die verkauften Produkte und die geleistete Arbeit permanent unter Druck steht. Gleichzeitig aber würde sich derselbe Durchschnittsbürger entschieden - und mit Recht - zur Wehr setzen, wenn er an seinem Arbeitsplatz durch eine Person aus Afrika oder Asien ersetzt würde, die bei gleichwertiger Qualifikation ein Gehalt bekäme, das dem Lohnniveau ihres Herkunftslandes entspricht. Weshalb diese Problematik auf derart unterschiedliche Weise wahrgenommen wird, ist unerklärlich und paradox zugleich, zumal es sich bei den zwei genannten Beispielen im Grunde um zwei Seiten derselben Medaille handelt.

Erwähnenswert ist auch, dass die Ausgaben für Nahrungsmittel gemessen an den Gesamtausgaben der Schweizer Haushalte im Laufe der Jahre stetig zurückgegangen sind: Lag ihr Anteil 1960 noch bei 27\%, fiel er während der 90er Jahre unter $10 \%$ und beträgt heute $7 \%$. Zwar erhöhten sich die Nahrungsmittelausgaben der Schweizer Konsumenten zwischen 1988 und 1998 absolut von 23 auf 27 Milliarden Franken. In der gleichen Zeitspanne jedoch verringerte sich jedoch der Anteil der Landwirte am Verkaufserlös von 38 auf 24\%, während jener des Zwischen- und des Detailhandels von 62 auf $76 \%$ anstieg. Die bedeutende Produktivitätssteigerung, welche die Landwirtschaft in dieser Zeit erzielte, wurde demnach vollumfänglich von den vor- und nachgelagerten Kettengliedern des Agrar- und Lebensmittelsektors absorbiert. Sollen künftig auch die Landwirte und andere Arbeitnehmer im Agrar- und Lebensmittelsektor wie zum Beispiel die landwirtschaftlichen Arbeiter oder die Angestellten der Grossverteiler für ihre Arbeit angemessen entlöhnt werden, so sind eine bessere Verteilung der Wertschöpfung in diesem Sektor und ein Anstieg der Lebensmittelkosten unverzichtbar ${ }^{4}$.

\section{IDEOLOGISCHER DRUCK VON INNEN}

Die neuesten strategischen Ansätze der Bundesbehörden zur künftigen Ausrichtung der Landwirtschaftspolitik lassen nichts Gutes erahnen. Derzeit wird unter dem Titel «Agrarpolitik 2007 » ein Entwurf zur Revision des Landwirtschaftsgesetzes und der Bestimmungen über den bäuerlichen Grundbesitz vorbereitet, dessen oberstes Ziel darin besteht, der Agrar- und Lebensmittelindustrie und dem Detailhandel Rohstoffe zu möglichst niedrigen Preisen zur Verfügung zu stellen. Um dieses Ziel zu erreichen, wird eine Strategie verfolgt, die auf der programmierten Beschleunigung des Restrukturierungsprozesses der Landwirtschaft beruht, sprich der schnellstmöglichen Liquidierung von 30 bis $40 \%$ der Schweizer Landwirtschaftsbetriebe, die als nicht wettbewerbsfähig und somit als langfristig nicht überlebensfähig gelten.

4. Bundesamt für Landwirtschaft, Agrarbericht 2000 und 2001, Bern. 
In dieser Form stellt das Projekt Agrarpolitik 2007 die ideologische Interpretation des verfassungsrechtlichen Konsens, der 1998 nach über zehnjähriger Debatte und verschiedenen Volksabstimmungen erzielt werden konnte, grundsätzlich in Frage. Indem die Wettbewerbsfähigkeit als oberstes Ziel definiert wird, dem alle anderen Aspekte unterzuordnen sind, wird gleichzeitig der Leistungsauftrag der Landwirtschaft in Frage gestellt. Die Autoren des Projekts verbergen nur mit Mühe ihr Misstrauen gegenüber den komplexen und vielfältigen Aufgaben, welche die Schweizer Landwirtschaft auf Grund des Artikels 104 der Bundesverfassung erfüllen muss ${ }^{5}$. Die Lancierung einer neuen Reform der Landwirtschaftspolitik knapp drei Jahre, nachdem mit der «Agrarpolitik 2002 » die letzte Reform in Kraft getreten ist, zeugt von einem äusserst überstürzten Vorgehen, welches das Schicksal zehntausender Bauernfamilien aufs Spiel setzt. Diese Überstürzung ist nicht etwa das Resultat internationalen Drucks, sondern eine zu 100\% hausgemachte «Strukturanpassung» und ein Abbau der Schweizer Landwirtschaft.

\section{EINE ANDERE VISION FÜR DIE ZUKUNFT}

Der künftige Bestand der Schweizer Landwirtschaft kann weder durch die Liquidierung von 30 bis $40 \%$ der Bauernbetriebe noch durch eine Überproduktion von Rohstoffen zu Spottpreisen gesichert werden. Es gilt vielmehr, eine möglichst hohe Zahl von Familienbetrieben verteilt auf das ganze Land zu erhalten, die Marktnähe der Landwirtschaft zu fördern, das Angebot (sprich : die produzierten Mengen) zu kontrollieren und qualitativ hoch stehende Produkte zu erzeugen. Nicht weniger wichtig sind Gewinn bringende, den Lebenskosten in der Schweiz entsprechende Verkaufspreise, neue Organisations- und Zusammenarbeitsformen unter den Produzenten, eine Balance im Kräfteverhältnis unter den Marktakteuren, die Stärkung der Beziehung zwischen Bauern und Konsumenten, die Bereitstellung einer breiten Palette von multifunktionalen Leistungen zu Gunsten der Allgemeinheit und eine Entschuldung der Landwirtschaftsbetriebe.

\section{DER DRUCK DER WTO}

Nicht alle Schweizer Bauern hegen in Bezug auf die WTO dieselben Befürchtungen, und auch ihre Forderungen gegenüber dieser Organisation sind längst nicht einheitlich. Einigkeit besteht jedoch in einem grundlegenden Punkt: Die sozialen, ökologischen, ethischen und kulturellen Dimensionen der Landwirtschaft wie auch ihr Beitrag zur Nahrungsmittelversorgung sind prioritär zu regeln, bevor die Liberalisierung des Agrarsektors weiter vorangetrieben wird. Neue WTO-Verhandlungen, die auf weitere Liberalisierungsschritte abzielen, bevor die anderen erwähnten Aspekte zufriedenstellend geregelt wurden, werden demnach auf breiten Widerstand stossen.

Die Westschweizer Bauerngewerkschaft Uniterre, die Mitglied der Coordination paysanne européenne und der internationalen Bewegung Via Campesina ist, lehnt eine neue Verhandlungsrunde ebenfalls ab und führt dabei weiter gehende Überlegungen an: Sie fordert nicht nur die ausdrückliche Anerkennung der

5. Vgl. Anhang 2. 
Multifunktionalität und der Nachhaltigkeit der Landwirtschaft, sondern auch der Souveränität der Länder im Hinblick auf die Nahrungsmittelversorgung. Es ist ein legitimes Recht der Staaten, autonom darüber zu entscheiden, wie sie die Nahrungsmittelversorgung für ihre Bevölkerung sicherstellen wollen, und sei es allenfalls durch eine verstärkte interne Stützung ihrer Landwirtschaft oder durch zusätzliche Schutzmassnahmen gegen aussen. Ferner lehnt Uniterre die Privatisierung der lebenden Ressourcen durch Patente und die Ausweitung der Kompetenzen der WTO, namentlich auf dem Gebiet des Service public, ab. Der Zugang zu Wasser, zu Saatgut, zur Bildung, zum Gesundheitswesen usw. müssen universelle Menschenrechte bleiben und dürfen nicht auf Dienstleistungen reduziert werden, die ausschliesslich jenen offen stehen, die sie sich leisten können.

Darüber hinaus fordert Uniterre eine tief greifende Reform der WTO und ihrer Entscheidungsmechanismen, bevor neue weltweite Deregulierungsschritte unternommen werden: Einbindung der WTO in das Netz der Sonderorganisationen der Vereinten Nationen, Neudefinition ihrer Zuständigkeiten und Integration der Welthandelsregeln in das bestehende völkerrechtliche Instrumentarium. Zudem sollten die derzeitigen und skandalösen Streitbeilegungsmechanismen der WTO schnellstmöglich durch einen unabhängigen internationalen Handelsgerichtshof ersetzt werden, denn unter den geltenden Verfahren können nach wie vor kollektive Wirtschaftssanktionen gegenüber Personengruppen, insbesondere Bauern, verhängt werden, die nicht im Mindesten an den eigentlichen Streitigkeiten beteiligt sind, wobei die Instanzen der WTO eine Doppelrolle als Richter und als Partei spielen.

\section{DIE LANDWIRTSCHAFT IN GESCHWÄCHTER POSITION}

Darüber hinaus sieht sich die Schweizer Landwirtschaft mit einer zweiten Problematik konfrontiert, die in internationalen Verhandlungen - bilateral oder im Rahmen der WTO - immer wieder zum Vorschein tritt : Der Beitrag der Landwirtschaft an die Wertschöpfung der Schweizer Wirtschaft ist im Laufe der Jahre kontinuierlich gesunken und lag Ende 1998 bei 1,1\% (4 Milliarden Franken von insgesamt 377 Milliarden). Selbst unter Berücksichtigung der Leistungen, die zu Gunsten der Allgemeinheit erbrachten werden und die jährlich mit rund 2 Milliarden Franken veranschlagt werden, beträgt der Anteil des Agrarsektors an der gesamten Volkswirtschaft des Landes knapp 1,5\%. Beim Aussenhandel betrugen die Einfuhren von Agrargütern 1999 6,8\% der gesamten Importe (8 Milliarden von insgesamt 120 Milliarden), während die Ausfuhren von Landwirtschaftsgütern 2,7\% der Gesamtexporte stellten (3,3 Milliarden von insgesamt 121 Milliarden) ${ }^{6}$.

Damit wird klar, dass die Interessen der Landwirtschaft im Rahmen internationaler Wirtschaftsverhandlungen für die Schweiz objektiv nicht prioritär sind. Es besteht Grund zur Befürchtung, dass die Interessen des Agrarsektors zu Gunsten der Anliegen stärkerer Wirtschaftszweige geopfert werden, dies umso mehr, als die Landwirtschaft der einzige Bereich ist, in dem die Schweiz sich mit aktiven Liberalisierungsmassnahmen zurückhält. 
Weiter gehende Konzessionen im Hinblick auf die Öffnung der Grenzen (Senkung der Importzölle und Erhöhung der Einfuhrkontingente) und auf den Abbau der internen Massnahmen zur Stützung der Landwirtschaft werden künftig in den Bauernkreisen auf entschlossenen Widerstand stossen. In der Tat geht es um das Überleben der Schweizer Landwirtschaft, die bereits heute jährlich 3 bis $4 \%$ ihrer Betriebe verliert. Zurzeit werden gerade noch rund 70'000 bewirtschaftete Bauernbetriebe gezählt. Durchaus legitim erscheint hingegen die langfristige Abkehr von Exportsubventionen in jenen Fällen, in denen sie Dumpingpraktiken auf den Exportmärkten gleichgestellt werden können. Aus schweizerischer Sicht betrifft dies hauptsächlich die Subventionierung des Exports von Hartkäse, der vornehmlich für die EU-Länder bestimmt ist. Diese Subventionen müssen im Rahmen der bilateralen Verträge zwischen der Schweiz und der EU innerhalb von 5 Jahren um mehr als $75 \%$ abgebaut werden.

\section{VOLLSTÄNDIGE LIBERALISIERUNG DER AGRARIMPORTE FÜR DIE 49 LDC?}

Der Bund schlägt vor, sämtliche Importzölle auf Agrargüter, die aus der Gruppe der 49 ärmsten Länder der Welt stammen (LDC: least developed countries, am wenigsten entwickelte Länder), schrittweise abzubauen. Damit schliesst sie sich einer ähnlich gelagerten Initiative der Europäischen Union mit dem Titel «Everything but Arms» an. Dabei sollen die Zölle in einem ersten Schritt bis zum 1. Januar 2002 um durchschnittlich $30 \%$ reduziert werden, Anfang 2004 um weitere $30 \%$ gesenkt und 2007 schliesslich vollständig aufgehoben werden. Die wichtigsten Argumente für diese Massnahme sind, dass die betreffenden Länder dadurch einen Nutzen ziehen könnten, dass sie die reichen Länder seit langem zu diesem Schritt aufgefordert hätten, dass im Rahmen der WTO entsprechende Vereinbarungen getroffen worden seien und dass es alles in allem schlicht egoistisch wäre, sich dieser Forderung zu widersetzen.

Aus dieser Perspektive betrachtet, scheint es in der Tat schwierig und vor allen Dingen unangebracht, einen Vorstoss zu torpedieren, der als Akt der Solidarität und als Massnahme zur Förderung des Gleichgewichts zwischen armen und reichen Ländern in den internationalen Beziehungen dargestellt wird. Geht man aber der Frage näher auf den Grund und versucht man zu eruieren, inwiefern sich eine vollständige Marktöffnung für Agrarprodukte aus den LDC auf die Länder des Nordens wie auch des Südens auswirken würde, präsentiert sich die Problematik in einem ganz anderen Licht. Der vorgeschlagene Liberalisierungsschritt gleicht einer unangebrachten, wenn nicht schädlichen medizinischen Therapie, bei der die Dosis systematisch erhöht wird, obwohl immer deutlicher wird, dass sich das Übel auf diese Weise nicht kurieren lässt.

\section{DIE VERLIERER: BAUERN IN DEN LDC ...}

Es gibt nicht den geringsten Beweis dafür, dass die ärmsten Länder einen realen Nutzen aus der Liberalisierung des Schweizer Agrarmarktes ziehen werden, geschweige denn, dass dieser hypothetische Nutzen den Bauern zugute kommen wird. Die Schweiz geht keinerlei Verpflichtung ein, wonach die Einfuhren in Zukunft auf der Basis Gewinn bringender und gerechter Preise erfolgen und so 
zu einer effektiven Verbesserung des Lebensstandards der Bevölkerung beitragen können, die davon abhängt. In anderen Worten, die Preise werden vom Weltmarkt diktiert. Diese Massnahme ist kein Akt der Solidarität, sondern höchstens eine Gelegenheit für die Schweiz, ihr etwas ramponiertes Image ohne erhebliche Kosten (einige Millionen nicht eingenommener Zölle) aufzupolieren und die wirtschaftlichen Folgen der Liberalisierung auf die Bauern in den Drittweltländern und in der Schweiz abzuwälzen, die auf der Verliererseite stehen werden.

Indem die Bauern der ärmsten Länder ermutigt werden, zu Weltmarktpreisen für den Export in die Schweiz zu produzieren, wird ein seit langem kritisierter Mechanismus im umgekehrten Sinn weitergeführt: die Ausfuhr der Agrarüberschüsse aus den Industrienationen in die ärmsten Länder der Welt, wodurch die Selbstversorgungswirtschaft jener Länder nachhaltig geschädigt wurde. Die einzigen Gewinner dürften höchstwahrscheinlich die international tätigen Unternehmen des Agrar- und Lebensmittelsektors sein, welche die Abschaffung der Zölle nutzen werden, um sich in den LDC niederzulassen, sich dort Ländereien anzueignen, Arbeiter zu äusserst niedrigen Löhnen zu beschäftigen und neue Märkte zu erobern - zum Schaden der Bauern im Süden wie im Norden.

Die Bauern in den südlichen Ländern sind sich dessen sehr wohl bewusst: Sie fordern deshalb in erster Linie die Entwicklung ihrer heimischen Märkte, den Schutz ihrer Landwirtschaft gegen das Dumping von Produktionsüberschüssen aus den Industrieländern, den Zugang zu Boden, Wasser und Saatgut und die Abschaffung der im Landwirtschaftsabkommen der WTO enthaltenen Klausel über den Mindestmartkzugang von 5\%. Der Vorstoss der Schweiz wird weder den Bedürfnissen noch den Forderungen der Bauern des Südens gerecht ${ }^{7}$.

\section{... UND IN DER SCHWEIZ}

Wird der Zugang zum Schweizer Markt für Agrargüter aus den 49 ärmsten Ländern an keine weiteren Bedingungen geknüpft, ist die Aufhebung der Einfuhrzölle nicht akzeptabel. In der Tat käme dies dem unlauteren Wettbewerb gegenüber einem oder mehreren wichtigen Agrargütern gleich, welche die Basis für das Einkommen der Schweizer Landwirte bilden (beispielsweise Zucker), und das Einkommen der Bauern in der Schweiz geriete unter erheblichen Druck. Zwar würde eine Schutzklausel eingeführt, um allzu negative Auswirkungen abzufedern, diese könnte aber den permanenten Preisdruck durch Güter, die zu Weltmarktpreisen eingeführt werden, nicht verhindern. Ferner steht zu befürchten, dass diese Massnahme nur ein Anfang ist und der freie Zugang zum Schweizer Markt in einem nächsten Schritt auf eine weitere Gruppe von Ländern ausgeweitet werden soll, die nicht zu den ärmsten Ländern der Welt gehören.

\section{MARKTZUGANG FÜR TROPISCHE ERZEUGNISSE BERECHTIGT}

Andererseits gewährt die Schweiz den LDC bereits heute den freien Marktzugang für fast alle tropischen Erzeugnisse, die für diese Länder naturgemäss die wichtigsten Produkte für den Export in Länder der gemässigten Klimazonen dar-

7. Vgl. Anhang 1 . 
stellen. In diesem Fall ist der Marktzugang vollkommen gerechtfertigt, da diese Erzeugnisse weder die einheimischen Produkte, noch die Schweizer Landwirtschaft gefährden - abgesehen von Absatzschwierigkeiten für einheimisches Obst, wenn zu gewissen Zeiten im Jahr gleichzeitig tropische Früchte viel zu billig angeboten werden (was wiederum ein sicheres Indiz für eine schlechte Entlöhnung der Bauern und Landarbeiter ist).

\section{WELCHE LÄNDER SIND BETROFFEN?}

Von den 49 ärmsten Länder der Welt befinden sich die Mehrzahl, nämlich 34, in Afrika und 7 in Asien, bei den restlichen handelt es sich um Inselstaaten in verschiedenen Regionen der Erde, darunter auch Haiti.

1999 führte die Schweiz aus diesen 49 Ländern Güter im Wert von 150 Millionen Franken ein. Allein zwei Drittel dieser Produkte stammten aus vier Ländern (Bangladesch, Liberia, Nepal und Uganda). Die Agrarprodukte machten einen Drittel der gesamten Importe aus diesen Staaten aus. Die typischen tropischen Erzeugnisse wie Kaffee, Tee oder Gewürze, die bereits heute ohne Einschränkungen in die Schweiz eingeführt werden können, sowie Tabak stellten zusammen mehr als $80 \%$ dieser Agrarimporte in Höhe von 50 Millionen Franken. Abgesehen von Tabak (der unter den bestehenden Importzöllen nicht sonderlich zu leiden scheint), kämen somit sämtliche anderen Produkte in den Genuss der Liberalisierung, darunter auch Milch und Milchprodukte, Fleisch, Zucker, Getreide und pflanzliche oder tierische Fette. Es handelt sich dabei - zumindest zu Beginn - um Güter im Wert von rund 10 Millionen Franken, was Zolleinnahmen von maximal einigen Millionen Franken entspricht.

\section{DAS BEISPIEL DES ZUCKERS}

Auf den ersten Blick erscheint die Bedrohung für die Schweizer Landwirtschaft sehr gering. Allerdings könnte sie sich rasch verschärfen. Dies lässt sich am Beispiel des Zuckers veranschaulichen: Der weltweite Zuckerverbrauch weist von Jahr zu Jahr nur geringe, hauptsächlich durch das Bevölkerungswachstum bedingte Schwankungen auf. Die Weltmarktpreise sind höchst volatil und liegen im Durchschnitt für fast alle Länder unter den Produktionskosten.

Der Schweizer Markt charakterisiert sich durch eine Steuerung des Angebots. Die inländische Zuckerproduktion ist kontingentiert und deckt mit rund 200'000 Tonnen etwa $65 \%$ des schweizerischen Verbrauchs ab. Die Preise werden direkt zwischen den Zuckermühlen und den Organisationen der Zuckerrübenproduzenten ausgehandelt. Damit ist dieser regulierte Markt vor Schwankungen und vor den unvorhersehbaren Entwicklungen auf dem Weltmarkt geschützt.

Mindestens neun der 49 LDC sind Zucker exportierende Länder (Birma, Kongo, Laos, Madagaskar, Malawi, Mosambik, Sambia, Sudan und Tansania). Die Zuckerausfuhren aus diesen Ländern sind mit insgesamt rund 350'000 Tonnen pro Jahr relativ gering. Die Zuckermühlen in diesen Ländern verfügen über genügend Kapazitäten, um beträchtlich höhere Zuckermengen zu verarbeiten. Eine Öffnung des schweizerischen Marktes ohne Mengenbeschränkung und 
Importzölle für die Einfuhr von Zucker aus den 49 LDC zu Weltmarktpreisen könnte sehr rasch zu einem Zusammenbruch des einheimischen Marktes führen, sofern keine angemessenen Schutzvorkehrungen getroffen werden, die über eine stets problematische und zeitlich begrenzte Schutzklausel hinausgehen. Will man sich gegenüber den LDC solidarisch zeigen und gleichzeitig den Schweizer Zuckerrübenproduzenten ein angemessenes Einkommen sichern, müssen weiter führende Massnahmen in Betracht gezogen werden. Die Lösung besteht in der Festlegung eines Kontingentes zu garantierten und Gewinn bringenden Preisen zu Gunsten der LDC. Diese Quote, die anfänglich einen Teil der derzeit gut 100'000 Tonnen importieren Zuckers abdeckt, könnte in regelmässigen Abständen erhöht werden. Auf diese Weise könnte sichergestellt werden, dass die Bauern in den LDC wie auch in der Schweiz von einer Initiative im Sinne des gerechten Handels profitieren können.

\section{FÜR ECHTE SOLIDARITÄT MIT DEN BAUERN DER 49 LDC}

Bereits heute ist der freie Zugang zum Schweizer Markt für Produkte aus den LDC, die nicht im direkten Wettbewerb mit den Erzeugnissen der Schweizer Landwirtschaft stehen, weitgehend gewährleistet. Bei einer Ausweitung des freien Marktzugangs auf Produkte, die auch in der Schweiz hergestellt werden, müssten folgende Regeln gelten:

a Festlegung von Importkontingenten zu garantierten Preisen zu Gunsten der 49 ärmsten Länder auf der Grundlage der derzeitigen Einfuhren.

- Anstatt die Importzölle einfach aufzuheben, sollte ein System zur Rückerstattung der Importzölle an die LDC errichtet werden. Eine Zweckbindung der zurückbezahlten Gelder sollte primär die angemessene Entlöhnung der Bauern, die Verbesserung der landwirtschaftlichen Infrastrukturen oder die Entschuldung der LDC sicherstellen.

- Im Gegenzug sollten die LDC die multifunktionellen Ziele der Landwirtschaft im Rahmen der WTO-Verhandlungen anerkennen und sich bereit erklären, Produkte zu liefern, die den minimalen Produktionsanforderungen entsprechen, welche auch von der Schweizer Landwirtschaft eingehalten werden müssen (Auflagen in Bezug auf Umwelt- und Pflanzenschutz u.a.m.).

- Bedingungslose Sicherstellung der Rückverfolgbarkeit und der Transparenz im Hinblick auf die Herkunft der eingeführten Güter, um dem Betrug und allfälligen Dreiecksgeschäften zwischen Drittstaaten und LDC vorzubeugen. In der Tat könnte es für andere Länder verlockend sein, die Herkunft von Agrargütern vorsätzlich falsch zu deklarieren, um die schweizerischen Importzölle zu umgehen.

口 Grundsätzlich wäre zu hinterfragen, ob es sinnvoll ist, tierische Produkte zu importieren, zu deren Erzeugung grosse Kalorien- und Futtermengen erforderlich sind, welche entscheidend zur Verbesserung der Ernährungslage der Menschen in den LDC beitragen könnten. 


\section{Anhang 1: Erklärung von Via Campesina und ROPPA*}

Via Campesina und das Netzwerk westafrikanischer Bauern- und Agrarproduzentenorganisationen (Réseau des organisations paysannes et de producteurs agricoles de l'Afrique de l'ouest, ROPPA) kritisieren die von der WTO befürwortete Liberalisierung des Handels mit Agrargütern und das Agrardumping der Exportmächte gegenüber Drittstaaten - vor allem gegenüber den am wenigsten entwickelten Ländern (LDC) welche die Produktionskapazitäten und die Ernährungsgewohnheiten empfindlich beeinträchtigen und sowohl im Norden als auch im Süden die bäuerliche Landwirtschaft verdrängen. So hat beispielsweise die Ausfuhr europäischer Agrarüberschüsse in die LDC zu Preisen, die weit unter dem in Europa oder in den LDC üblichen Niveau liegen, zu einem Zusammenbruch der Agrarproduktion in diesen Ländern geführt.

Der Beschluss der Europäischen Union, ihren Agrarmarkt für Produkte aus den LCD zu öffnen und auf Importzölle zu verzichten, läuft den wahren Bedürfnissen dieser Länder zuwider, denn anstatt den Bauern der LDC eine echte Chance zu geben, ihre Produkte in Europa abzusetzen, soll damit in erster Linie die Erschliessung der Märkte in den LDC durch EU-Exporteure gerechtfertigt werden.

Via Campesina und ROPPA sind der Ansicht, dass der Aufbau einer Selbstversorgungswirtschaft, die gesunde, qualitativ hoch stehende und den kulturellen Gebräuchen entsprechende Produkte für den Binnenmarkt der einzelnen Länder und für den subregionalen oder regionalen Markt in den einzelnen Erdteilen herstellt, Priorität besitzen muss.

Die Bauern in den LDC wollen in erster Linie für sich selbst und für ihre Familien produzieren, bevor sie Güter für den Binnenmarkt herstellen - vom Export ganz zu schweigen. Der Beschluss der EU trägt dazu bei, die Gewinne der Grossunternehmen zu steigern, die mit billigen Arbeitskräften in den LDC Güter herstellen, die für den Export nach Europa bestimmt sind. Dadurch werden der Nahrungsmittelproduktion für die Bauern und die Stadtbevölkerung in diesen Ländern dringend benötigte Ressourcen und Arbeitskräfte entzogen und die Ernährungslage verschlechtert.

Via Campesina und ROPPA bekräftigen folgende Grundsätze:

- das Recht der Bevölkerungen und ihrer Regierungen, ihre Agrar- und Lebensmittelpolitik auf nationaler und regionaler Ebene selbst bestimmen zu können und damit die landwirtschaftlichen Familienbetriebe zu unterstützen, vorausgesetzt, diese Politiken sehen von Dumping-Praktiken gegenüber Drittstaaten ab (Souveränität der Länder im Hinblick auf die Nahrungsmittelversorgung);

• das Recht auf Zugang zu Ressourcen (Land, Wasser, Saatgut, usw.), um die Produktion sicherzustellen und angemessen leben zu können, denn den Bauern in den Ländern des Südens werden diese Rechte nach wie vor verwehrt;

口 das Recht der Länder des Südens wie des Nordens, allein oder gemeinsam ihre Landwirtschaft und ihre Märkte zu schützen und so ein angemessenes Einkommen der bäuerlichen Familienbetriebe sicherzustellen und gerechte Preise für ihre Erzeugnisse zu gewährleisten;

- der Weltmarkt darf nicht mehr ein Markt der Produktionsüberschüsse sein, sondern ein Ort des gerechten Austauschs für Produkte aus verschiedenen Ländern, auf dem angemessene Preise erzielt werden können. Die internationalen Märkte müssen reguliert werden, um der kontinuierlichen Verschlechterung der Handelsbedingungen, insbesondere für die Bauern aus den Ländern des Südens, welche tropische Erzeugnisse exportieren, ein Ende zu setzen.

Via Campesina und ROPPA fordern die Regierungen des Nordens wie auch des Südens auf,

- Agrarpolitiken zu verfolgen oder Reformen durchzuführen, welche das Überleben der bäuerlichen Familienbetriebe sichern;

口 keine internationalen Zusammenarbeitsformen zu unterstützen, welche an Liberalisierungs- und Privatisierungsverpflichtungen gekoppelt sind, die den Prioritäten der einzelnen Länder zuwiderlaufen;

口 dem Aufbau der Binnenmärkte (auf lokaler, nationaler und regionaler Ebene) gegenüber dem Export Priorität einzuräumen;

口 den Erwartungen der Bevölkerung in Bezug auf die Ernährung, die weiteren Grundbedürfnisse und die Umwelt gegenüber den Interessen der multinationalen Konzerne Vorrang zu geben;

a die Patentierung lebender Ressourcen sowie die Produktion und die Einfuhr gentechnisch veränderter Organismen (GVO) zu verbieten.

* Übersetzung IUED. 


\section{Anhang 2: Bundesverfassung}

Art. 104 Landwirtschaft

1 Der Bund sorgt dafür, dass die Landwirtschaft durch eine nachhaltige und auf den Markt ausgerichtete Produktion einen wesentlichen Beitrag leistet zur:

a. sicheren Versorgung der Bevölkerung;

b. Erhaltung der natürlichen Lebensgrundlagen und zur Pflege der Kulturlandschaft;

c. dezentralen Besiedlung des Landes.

${ }^{2}$ Ergänzend zur zumutbaren Selbsthilfe der Landwirtschaft und nötigenfalls abweichend vom Grundsatz der Wirtschaftsfreiheit fördert der Bund die bodenbewirtschaftenden bäuerlichen Betriebe.

${ }^{3}$ Er richtet die Massnahmen so aus, dass die Landwirtschaft ihre multifunktionalen Aufgaben erfüllt. Er hat insbesondere folgende Befugnisse und Aufgaben:

a. Er ergänzt das bäuerliche Einkommen durch Direktzahlungen zur Erzielung eines angemessenen Entgelts für die erbrachten Leistungen, unter der Voraussetzung eines ökologischen Leistungsnachweises.

b. Er fördert mit wirtschaftlichen Anreizen Produktionsformen, die besonders naturnah, umwelt- und tierfreundlich sind.

c. Er erlässt Vorschriften zur Deklaration von Herkunft, Qualität, Produktionsmethode und Verarbeitungsverfahren für Lebensmittel.

d. Er schützt die Umwelt vor Beeinträchtigungen durch überhöhten Einsatz von Düngstoffen, Chemikalien und anderen Hilfsstoffen.

e. Er kann die landwirtschaftliche Forschung, Beratung und Ausbildung fördern sowie Investitionshilfen leisten.

f. Er kann Vorschriften zur Festigung des bäuerlichen Grundbesitzes erlassen.

${ }^{4}$ Er setzt dafür zweckgebundene Mittel aus dem Bereich der Landwirtschaft und allgemeine Bundesmittel ein. 